


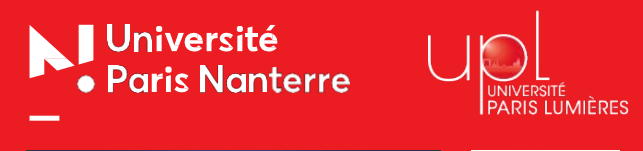

-

(TAD

Centre de Theorie et Analyse du Droit

200 av. de la République

www.parisnanterre.fr

\title{
Entre inclusion et
}

\section{exclusion. \\ La double face de la \\ citoyenneté}

\section{JEUDI 24 \& VENDREDI 25 MARS 2022 En salle des Conférences (Bât. Formation Continue)}

\author{
Inscription (libre) obligatoire sur : https://forms.gle/R9aF31Vu1svYhKpY9
}

II est généralement soutenu qu'en tant qu'elle désigne une appartenance, la citoyenneté est « constitutivement orientée en fonction d'un intérieur et d'un extérieur, de l'inclusion aussi bien que de l'exclusion » (D. Gösewinkel). Elle « requiert et produit nécessairement, comme son double inversé, le non-citoyen ".

Contrairement à la présentation souvent retenue, ce non-citoyen n'est pas seulement l'étranger. La nationalité n'est pas une condition suffisante de la citoyenneté. Son accès est aussi subordonné à une exigence d'autonomie ou d'indépendance et de dignité qui rend compte de l'existence de nationaux noncitoyens.

Cette seconde déclinaison de l'opposition entre un « intérieur » et un « extérieur » de la citoyenneté, au sein même des frontières de la nation, est remise en cause ces dernières années. Le droit de vote a été accordé sinon totalement, du moins en grande partie aux deux dernières figures de l'exclu que sont le condamné et l'« incapable ».

II est remarquable que le législateur et le juge aient justifié leur inclusion en appliquant pour la première fois en ces termes aux droits politiques des principes importés du droit des droits de l'homme : le principe de non-discrimination et le principe de dignité de la personne. En vertu de ces fondements nouveaux, la citoyenneté devrait logiquement être étendue aux étrangers.
Cette conclusion reste toutefois fort discutée. Pour certains, « I'extériorité de l'étranger serait d'un type particulier : il serait l'exclu dont la citoyenneté démocratique a besoin pour pouvoir être comprise comme une appartenance » (C. Colliot-Thélène).

\section{L'objet de ce colloque consiste à examiner} cette tension qui traverse la citoyenneté, entre l'appartenance à la communauté politique, qui est nécessairement particulière (dimension exclusive) et l'universalisme des droits du citoyen (dimension inclusive).

On se demandera si cette tension - qui prend une dimension nouvelle aujourd'hui que le droit positif tend à rapprocher toujours plus les figures du citoyen et de l'homme - est constitutive de la citoyenneté ou si elle peut être dépassée.

Afin d'y répondre, on s'intéressera aussi bien au droit existant (à la citoyenneté dans l'Union européenne par exemple) qu'aux discours qui mobilisent la notion de citoyenneté pour décrire des modes non classiques de construction d'un rapport au politique, dans lesquels la participation est déliée des conditions auxquelles elle est traditionnellement liée. 


\section{JEUDI 24 MARS 2022}

\section{Matinée | de 9h à $12 \mathrm{~h} 40$}

Présidence : Jean-Louis Halpérin (ENS PSL)

\section{Citoyenneté et particularisme de l'appartenance - ou la dimension exclusive de la citoyenneté}

9h | Accueil des participants et ouverture du colloque

Mots d'accueil

Par Véronique Champeil-Desplats, Vice-Présidente déléguée à la recherche de I'Université Paris Nanterre et membre de la ComUE Université Paris Lumières

Au nom du CTAD et de l'équipe CREDOF

Propos introductifs par Camille Aynès

9h30 | La citoyenneté comme dignitas en droit romain : (dé)-classer et/ou exclure?

Clément Bur (INU Champollion Albi - PLH-ERASME Toulouse 2)

9h55 | Citoyenneté et nationalité, deux types d'appartenance distincts ?

Jules Lepoutre (Université de Corse)

10h20 | Quelle citoyenneté pour les femmes? L'exclusion par l'application du principe d'égalité Elsa Fondimare (Université Paris Nanterre)

10h45 | Échanges avec la salle

11h15 | Pause

$11 \mathrm{~h} 30$ | Les États-Unis, une démocratie où le droit de vote n'est pas garanti ? La question du « vote noir » dans l'histoire constitutionnelle américaine

Cécile Guérin-Bargues (Université Panthéon-Assas)

11h55 | L'appartenance saisie par le droit

Danièle Lochak (Université Paris Nanterre, émérite)

12h20 | Échanges avec la salle

12h40 | DÉJEUNER

\section{Après-midi | de 14h15 à $17 \mathrm{~h} 30$}

Présidence : Thomas Hochmann (Université Paris Nanterre)

\section{Citoyenneté et universalisme des droits - ou la dimension inclusive de la citoyenneté}

14h15 | Citoyenneté, non-discrimination et dignité de la personne : l'inclusion des indignes et des incapables

Camille Aynès (Université Paris Nanterre/ComUE Paris Lumières) 
14h40 | La dynamique constitutionnelle de la citoyenneté inclusive en droit comparé Jordane Arlettaz (Université de Montpellier)

15h05 | Citoyenneté et personnalité des robots autonomes Serge Slama (Université Grenoble-Alpes)

15h30 | Échanges avec la salle

16h | Pause

16h15 | Réflexions sur l'intitulé initial de la loi sur le respect des principes républicains, dite Loi contre le séparatisme et « les atteintes à la citoyenneté »

Valentin Gazagne-Jammes (Université de Bordeaux)

16h40 | La citoyenneté en temps de crise

François Saint-Bonnet (Université Panthéon-Assas)

17h05 | Échanges avec la salle

\section{VENDREDI 25 MARS 2022}

\section{Matinée | de 9h15 à $12 \mathrm{~h} 30$}

\section{Présidence : Véronique Champeil-Desplats (Université Paris Nanterre) \\ Une tension constitutive de la citoyenneté ?}

9h15 | Citoyenneté de l'Union européenne et nationalité des États-membres Myriam Benlolo-Carabot (Université Paris Nanterre)

9h40 | Citoyenneté européenne et "valeurs de droite " (sécurité, autorité, forme de vie) Loïc Azoulai (Sciences Po Paris)

10h05 | Échanges avec la salle

10h25 | Pause

10h40 | Le critère de résidence, facteur de marginalisation dans l'appréhension de la citoyenneté : le cas des gens du voyage Marthe Fatin-Rouge Stefanini (Université Aix-Marseille)

11h05 | Citoyenneté et mobilité : l'exercice de la citoyenneté par les citoyens installés à l'étranger Anne-Sophie Traversac (Université Panthéon-Assas)

$11 \mathrm{~h} 30$ | Un droit de citoyenneté mondial : cette notion a-t-elle un sens ?

Catherine Colliot-Thélène (Université Rennes I, émérite)

11h55| Échanges avec la salle

12h25| DÉJEUNER 
Présidence : Olivier Beaud (Université Panthéon-Assas)

\section{Une tension dépassable au prix d'une reconceptualisation de la citoyenneté ?}

14h| De quelle société politique le citoyen numérique est-il membre?

Pauline Türk (Université Côte d'Azur)

14h25| Le CESE et sa réforme : de quelle citoyenneté parle-t-on?

Julien Padovani (Université Paris 1)

14h50| La citoyenneté, une création constitutionnelle continue

Dominique Rousseau (Université Paris 1, émérite)

15h15 Échanges avec la salle

15h45| Pause

16h| Y a-t-il des formes de démocratie plus démocratiques que les autres?

Table ronde avec Éric Buge (Administrateur de l'Assemblée nationale), Bruno Daugeron (Université Paris Descartes) et Arnaud le Pillouer (Université Paris Nanterre)

16h45| Propos conclusifs

17h| Clôture du colloque

\section{Plan du campus de Nanterre - Université Paris Nanterre}

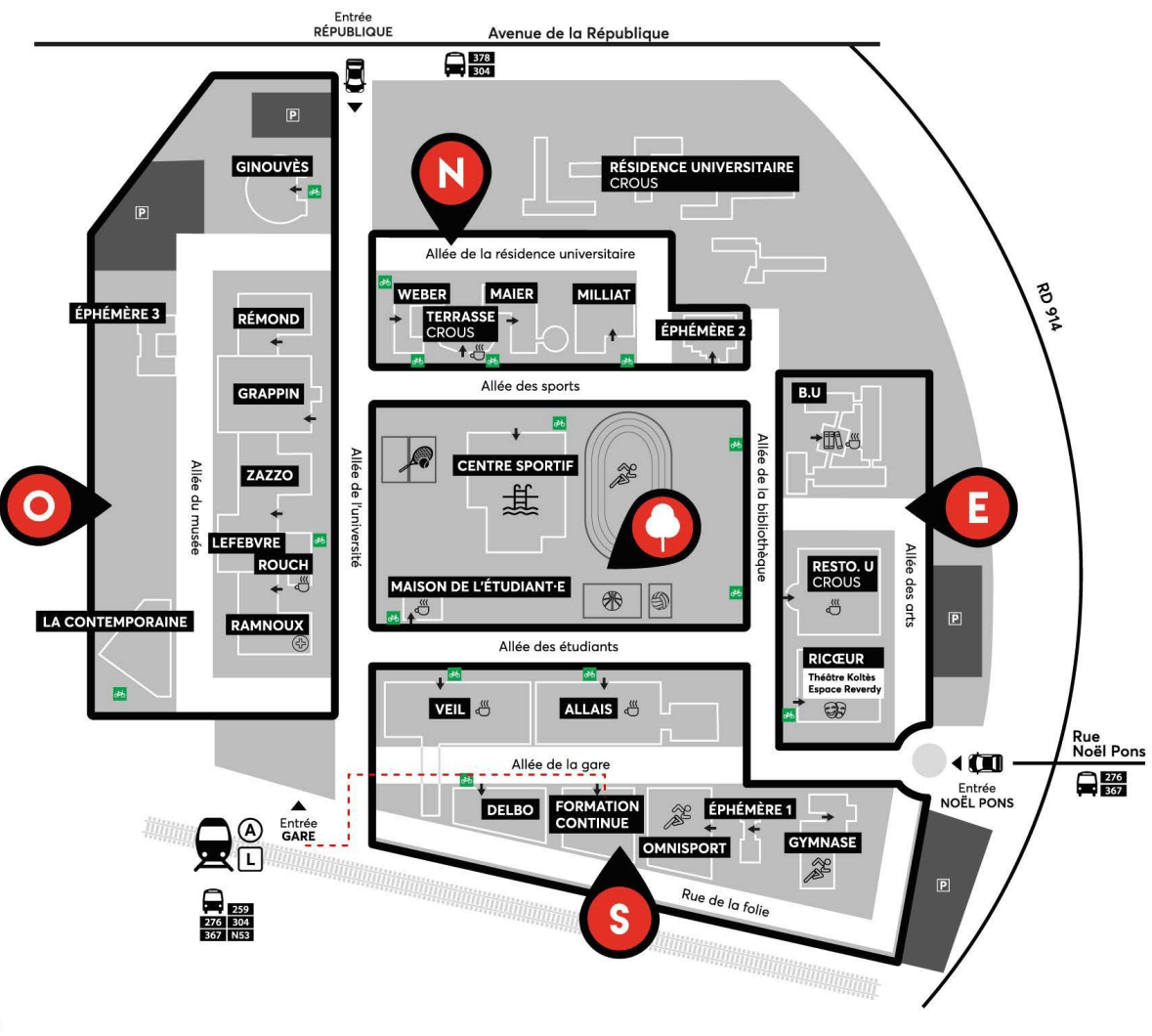

Contact et informations: mbdiallo@parisnanterre.fr 